\section{Topical nitrates potentiate the effect of botulinum toxin in the treatment of patients with refractory anal fissure}

\author{
J Lysy, Y Israelit-Yatzkan, M Sestiery-Ittah, S Weksler-Zangen, D Keret, E Goldin
}

This treatment modality appears to be safe and promising. (2) ID application induced a greater reduction in MARP following BT injection compared with ID application before BT injection. The improved potency of ID on MARP after BT injection suggests a primary cholinergic tonus dominance in some patients and not, as previously claimed, anal sphincter insensitivity to nitrates.

(Gut 2001;48:221-224)

Keywords: nitrates; botulinum toxin; anal fissure

therapeutic effect and lowering action on internal anal sphincter pressure of BT injection and local application of isosorbide dinitrate (ID) compared with BT given alone, in patients with chronic anal fissure (CAF) refractory to treatment with ID.

Methods-Thirty consecutive patients with CAF who did not respond to previous topical ID treatments were randomly assigned to receive one of the following treatments: group A, injection of BT (20 U into the internal anal sphincter) and subsequent daily applications of ID (2.5 mg three times daily for three months); and group B, BT injection only $(20 \mathrm{U})$. If at the end of six weeks following BT injection no improvement was seen in group B, ID was added. A series of anal pressure measurements, including resting basal pressure and resting pressure following topical ID (1.25, 2.5, and $3.75 \mathrm{mg}$ ), was carried out both before and two weeks after $20 \mathrm{U}$ of BT injection into the internal anal sphincter. At the end of the trial, patients were followed up for an average period of 10 months.

Findings-At six weeks the fissure healing rate was significantly higher in group $A$ patients $(10 / 15(66 \%))$ compared with group $B(3 / 15(20 \%))(p=0.025)$. At eight and 12 weeks, no significant differences were seen: $11 / 15(73 \%) v 11 / 15(73 \%)$ and $9 / 15(60 \%) v 10 / 15(66 \%)$, group A $v$ group $B$, respectively. Maximum anal resting pressure (MARP) was significantly lower two weeks after BT injection than baseline MARP (90 (4) $v 110$ (5) mm Hg; p<0.001). A significantly greater reduction in MARP following local application of ID was achieved after BT injection compared with that achieved before BT injection $(\mathrm{p}=\mathbf{0 . 0 3 7})$

Interpretation-(1) Combined BT injection and local application of ID in patients with CAF who failed previous treatment with ID was more effective than BT alone.
Anal fissure is a painful longitudinal or elliptical ulcer in the anoderm, extending from below the dentate line to the anal verge. In patients with fissures, basal anal pressure is significantly elevated. ${ }^{1}$

Current evidence suggests that idiopathic anal fissures are caused by high internal anal sphincter (IAS) pressure and secondary local ischaemia. ${ }^{2-4}$ Acute anal fissure heals spontaneously or with conservative treatment within six weeks, whereas chronic anal fissure is more intractable and is unlikely to heal with conventional conservative management. ${ }^{5}$ Surgical mended treatment. However, the procedure may be associated with permanent impairment of continence. ${ }^{6}$ In vitro and in vivo studies in animals have established that nitric oxide (NO) is probably the most important inhibitory neurotransmitter in the IAS. ${ }^{7}$ Evidence suggests that organic nitrates such as isosorbide dinitrate (ID) and glyceryl trinitrate are converted to NO by a common enzyme. ${ }^{8}$ Glyceryl trinitrate cream applied locally to the anus has been shown to cause lowering of IAS pressure in healthy subjects ${ }^{9}$ and to promote healing of anal fissures. ${ }^{10-12}$ The other pharmacological approach involves the use of botulinum toxin (BT). Again, the aim is to decrease resting anal pressure, in this case by preventing release of acetylcholine from presynaptic nerve terminals. Maria et al reported a $73 \%$ healing rate two months after BT injection into the IAS. ${ }^{13}$ BT and local nitrates lower resting pressure by different mechanisms: we therefore decided to use them simultaneously in the treatment of patients with refractory chronic anal fissure (CAF). The aims of the present study were to

Abbreviations used in this paper: $\mathrm{BT}$, botulinum toxin; NO, nitric oxide; IAS, internal anal sphincter; ID, isosorbide dinitrate; CAF, chronic anal fissure; MARP, maximum anal resting pressure. reduction of resting pressure is the recom- 
treat patients with CAF, refractory to treatment with ID, by injection of BT in combination with local application of ID compared with injection of BT alone. We assessed both the therapeutic effects and lowering of IAS pressure of the two treatments.

\section{Patients and methods}

Thirty consecutive patients with CAF who did not respond to previous topical ID treatment given over a period of at least six weeks were recruited to participate in the study. Informed consent was obtained from each patient. At study initiation, anal manometry was performed in all patients using a constantly perfused side hole catheter (Sandhill TDS 8000, Highlands-Ranch, Colorado, USA). Basal maximum anal resting pressure (MARP) was recorded 10 minutes after insertion of the catheter. MARP was recorded again $10 \mathrm{~min}$ utes after the first dose of $1.25 \mathrm{mg}$ ID to the anus. A further dose of $1.25 \mathrm{mg}$ was then applied. We allowed 10 minutes for stabilisation at each dose increment, and manometric studies were repeated up to a total dose of 3.75 mg ID. We used Isoket spray (SchwartzPharma, Germany), which delivers $1.25 \mathrm{mg}$ of ID at each pressure. Squeeze pressure was also recorded. All manometric studies were performed at least 24 hours after cessation of local application of nitrates. At this stage type A botulinum toxin (Botox, Allergan, Ireland) was diluted in saline to $40 \mathrm{U} / \mathrm{ml}$ : $10 \mathrm{U}$ of dilute BT was injected with a 27 gauge needle into the IAS on both sides lateral to the fissure (total dose $20 \mathrm{U}$ ) in all patients.

Patients were randomly divided into two treatment groups: group A, BT injection and subsequent daily applications of ID spray (2.5 mg three times daily) for three months; and group B, BT injection only. If at the end of six weeks following BT injection, no improvement was seen in group B patients, ID was added as in group $\mathrm{A}$.

Following BT injection, all patients were evaluated at two week intervals for a period of three months. All patients were reexamined and questioned about side effects and level of pain experienced on defecation. On the first follow up visit, two weeks after BT injection, the same manometric studies as described above were repeated. Thereafter, the healed patients were contacted by telephone every three months and were encouraged to keep in contact if symptoms recurred.

STATISTICAL STUDIES

Age, symptoms duration, and time to healing are expressed as mean (SEM). Variables in groups A and B were compared using the Student's $t$ test and non-parametric MannWhitney test, when appropriate. Fisher's exact test was used to compare the success rate between groups A and B. The effect of $3.75 \mathrm{mg}$ of ID on the decrease in MARP was tested before and after BT injection using a matched pairs two sided $t$ test.

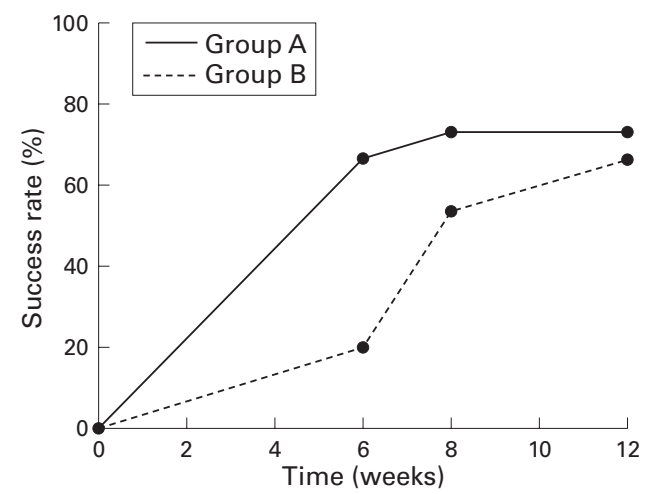

Figure 1 Progress of healing in groups $A$ and $B$.

\section{Results}

BASELINE ASSESSMENT

Group A comprised 15 patients (six men, nine women), mean age 43.9 (3) years (range 21-67). Symptoms had been present for a mean period of 18.2 (3) months. Group B also comprised 15 patients (six men, nine women), mean age 45.3 (4) years (range 24-72). Symptoms had been present for a mean period of 21.1 (3) months. MARP was 109 (6) $\mathrm{mm} \mathrm{Hg}$ in group A and 109 (7) $\mathrm{mm} \mathrm{Hg}$ in group B (NS). The maximum voluntary squeeze pressure in group A was 89.1 (9) $\mathrm{mm} \mathrm{Hg}$ and in group B 80.5 (9) mm Hg (NS) .

\section{FISSURE RESPONSE}

At six weeks, the fissure healing rate was significantly higher in group A patients: 10/15 $(66 \%) v 3 / 15(20 \%)$ in group B $(p=0.025)$. At six weeks, ID was added to the non-responders of group B, and at eight and 12 weeks no significant differences were seen: 11/15 (73\%) v $9 / 15(60 \%)$ and $11 / 15(73 \%)$ v $10 / 15(66 \%)$ (group A $v$ group B, respectively) (fig 1). Mean time for pain relief was 11.4 (2) days in group A and 18.3 (5) days in group B (NS). During follow up, one patient in each group relapsed; this occurred four and six weeks after healing. Three patients who had not healed at three months received a second BT injection of $25 \mathrm{U}$ : this was also unsuccessful and those patients then underwent lateral sphincterotomy. The other treatment failures refused to be operated on because they were symptom free or only mildly symptomatic. Side effects occurred in one patient who had perianal thrombosis following BT injection and two patients had mild transient headache following ID application.

MANOMETRIC STUDIES

MARP was significantly reduced two weeks after BT injection: 24.2 (4)\% in group A and 20.6 (2)\% in group B (NS). The pressure decreased in both groups from 110 (5) $\mathrm{mm} \mathrm{Hg}$ before injection to 90 (4) $\mathrm{mm} \mathrm{Hg}$ after injection $(p<0.001)$. Significantly greater reductions in MARP following different doses of topical ID were achieved after BT injection compared with the reductions before BT injection ( $\mathrm{p}=0.037)$ (fig 2). Maximum voluntary pressure decreased by 4.8 (1)\% and 4.4 (1)\% in groups A and B, respectively (NS). 


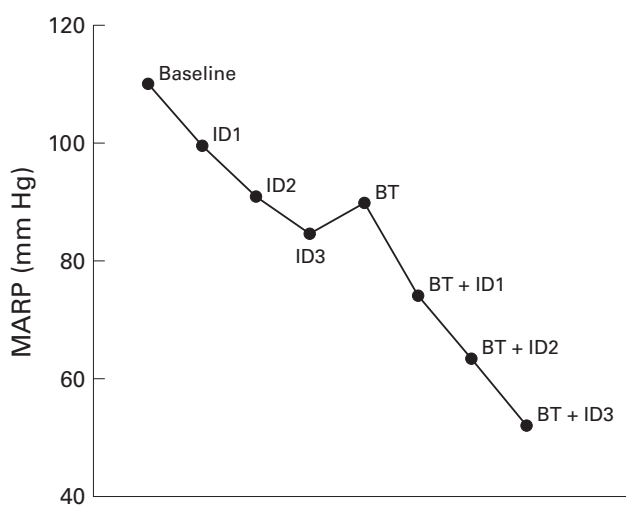

Figure 2 Decrease in maximum anal resting pressure (MARP) by isosorbide dinitrate (ID) and botulinum toxin (BT) in refractory anal fissure patients. Baseline shows mean internal anal sphincter (IAS) pressure in all fissure patients in the study before treatment. ID1, ID2, and ID3 indicate decreases in IAS pressures after 1.25, 2.50 and $3.75 \mathrm{mg}$ of ID before any treatment. BT indicates mean IAS pressure in all study patients two weeks after BT injection. ID $+B T$ indicates decrease in pressure with increasing dose of ID two weeks after BT injection.

\section{Discussion}

Local nitrates can assist anal healing by reducing anal pressure and inducing local vasodilatation. BT relaxes the anal sphincter by a mechanism different from that of local nitrates: therefore, we decided to administer BT to those patients who had failed treatment with ID. The first purpose of the study was to compare the therapeutic efficacy of combined treatment with BT and ID with that of BT alone. At six weeks, the fissure healing rate was significantly higher in patients who received the combined treatment compared with patients treated with BT alone. At six weeks, ID was added to those patients treated with BT alone who failed to heal. At eight and 12 weeks, no significant difference was seen between groups. Hence addition of topical ID at six weeks to non-responders (or very slow responders) in group B induced faster fissure healing.

The overall success rate in both groups at the end of the study was $70 \%$ which is low compared with our previous study with ID alone $^{14}$ and other studies where BT was injected. ${ }^{131516}$ Hence the success rate in the present study using the combined drug treatment seems to be relatively low compared with the success rate reported in previous studies using single drug treatment. This may be explained by the refractory nature of the fissures in the present study which were previously resistant to the therapeutic effect of local nitrates alone. In addition, the majority of our patients had fissures with the classic chronic appearance (abundant scar tissue and poor blood perfusion) which probably prevented faster and more effective fissure healing. This argument may also explain the relatively long time required to achieve pain relief, which was 14 (3) days in both groups (compared with 6.5 days with ID alone in the previous $s t u d y^{14}$ ). Side effects were rare. Only two patients complained of mild transient headache following ID application; this low rate of headache can be explained by the long term prestudy ID treatment and tolerance acquired to this effect of the drug. In contrast, drug tolerance was not a problem in reducing IAS pressure. One patient had perianal thrombosis following BT injection. Incontinence problems were not reported by any patient despite very low MARP levels (sometimes to $30 \mathrm{~mm} \mathrm{Hg}$ ) induced by the treatment. This can be explained by the relatively young age of our patients and their well preserved continence.

The second purpose of the study was to evaluate the lowering effect on MARP of either BT alone or ID application before and after BT injection. The mean decrease in MARP following $2.5 \mathrm{mg}$ ID alone was $17.2 \%$ : this value is lower than that achieved in the success group in our previous study (32\%) and is similar to the failure group in that study $(16 \%) .{ }^{14}$ Following BT injection alone, MARP decreased significantly. When ID application was given after BT, MARP decreased significantly more than when ID was given before BT injection (see fig 2).

No significant differences were found when baseline MARP or its percentage decrease following treatment were compared between responders and non-responders. However, chronic fissure changes were more prominent in non-responders, suggesting that abundant fibrous tissue is an important factor that may negatively influence fissure healing despite significant pressure reduction rates.

Watson et al found that patients with CAF who did not respond to nitrate treatment had a smaller reduction in pressure following nitrate application compared with responders and healthy controls. They concluded that the pathogenesis of CAF and the cause of the failure to heal following nitrate treatment is related, at least in part, to an insensitivity of the IAS to endogenous and exogenous NO, respectively. ${ }^{11}{ }^{12}$ However, our results suggest a primary cholinergic tonus dominance in some patients as the relaxing effect of NO could be fully expressed following cholinergic block with BT.

We conclude that ID potentiates the therapeutic effect of BT in patients with refractory chronic anal fissure. The significantly improved pressure lowering effect of ID following BT injection compared with its lowering effect before BT injection suggests a primary cholinergic tonus dominance in patients with refractory anal fissure.

The authors thank Ms Aviva Bookin, Gastroenterology Unit, Hadassah University Hospital, for help in writing the manuscript and Ms Ruth Feuer for technical assistance.

1 Farouk R, Duthie G S, MacGregor A, et al. Sustained internal sphincter hypertonia in patients with chronic anal fissure. Dis Colon Rectum 1994;37:424-9.

2 Greenfield LJ, Mulholland MW, Oldhamt KT, et al. Surgery scientific principles and practice. Philadelphia: JB Lippincott, 1993:1071-3

3 Klosterhalfen B, Vogel P, Rixen H, et al. Topography of the nferior rectal artery: a possible cause of chronic primary anal fissure. Dis Colon Rectum 1989;32:43-52.

4 Schouten WR, Briel JW, Auwerda JJ. Relationship between anal pressure and anoderm blood flow: the vascular pathogenesis of anal fissure. Dis Colon Rectum 1994;37:664-9.

5 Fleshman JW. Anorectal motor physiology and pathophysiology. Surg Clin 1993;73:1245-65.

6 Vafai M, Mann CV. Closed lateral internal anal sphincterotomy as an office procedure for the treatment of anal fisotomy as an office procedure for
sure. Colon Proctol 1987;9:47-53.

7 Chakder S, Rattan S. Release of nitric oxide by activation of nonadrenergic noncholinergic neurons of internal anal sphincter. Am f Physiol 1993;264:G7-12. 
8 Chung SJ, Fung HL. A common enzyme may be responsible for the conversion of organic nitrates to nitric oxide in vascular microsomes. Bichem Biopsy Res Commun 1992;30 932-7.

9 Loder PB, Kamm MA, Nicholls RJ, et al. Reversible chemical sphincterotomy by local application of glyceryle trinitrate. Br F Surg 1994;81:1386-9.

10 Gorfine SR. Treatment of benign anal disease with topical nitroglycerine. Dis Colon Rectum 1995;38:453-7.

11 Watson SJ, Kamm MA, Nicholls RJ, et al. Topical glyceryl trinitrate in the treatment of chronic anal fissure. Br $\mathcal{F}$ Surg 1996;83:771-5.

12 Lund JN, Armitage NC, Scholefield JH. Use of glyceryl trinitrate ointment in the treatment of anal fissure. $\mathrm{Br} F$ Surg 1996;83:776-7.
13 Maria G, Cassetta, Gui D, et al. A comparison of botulinum toxin and saline for the treatment of chronic anal fissure. $N$ Engl f Med 1998;338:217-20

14 Lysy J, Israelit-Yatzkan Y, Sestiery-Ittah M, et al. Treatment of chronic anal fissure with isosorbide dinitrate: long term results and dose determination. Dis Colon Rectum 1998;41: 1406-10.

15 Jost WH. One hundred cases of anal fissure treated with botulinum toxin: early and long term results. Dis Colon Rectum 1997;40:1029-32.

16 Brisinda G, Maria G, Bentivoglio AR, et al. A comparison of injection of botulinum toxin and topical nitroglycerin ointment for the treatment of chronic anal fissure. $\mathrm{N} \mathrm{Engl} \mathcal{F}$ Med 1999;341:65-9. 